\title{
QUASI-REPRESENTATIONS OF FINSLER MODULES OVER $C^{*}$-ALGEBRAS
}

\author{
M. AMYARI ${ }^{1 *}$, M. CHAKOSHI ${ }^{1}$ AND M. S. MOSLEHIAN ${ }^{2}$
}

\begin{abstract}
We show that every Finsler module over a $C^{*}$-algebra has a quasi-representation into the Banach space $\mathbb{B}(\mathscr{H}, \mathscr{K})$ of all bounded linear operators between some Hilbert spaces $\mathscr{H}$ and $\mathscr{K}$. We define the notion of completely positive $\varphi$-morphism and establish a Stinespring type theorem in the framework of Finsler modules over $C^{*}$-algebras. We also investigate the nondegeneracy and the irreducibility of quasi-representations.
\end{abstract}

\section{INTRODUCTION}

The notation of Finsler module is an interesting generalization of that of Hilbert $C^{*}$ module. It is a useful tool in the operator theory and the theory of operator algebras and may be served as a noncommutative version of the concept of Banach bundle, which is an essential concept in the Finsler geometry. In 1995 Phillips and Weaver [10] showed that if a $C^{*}$-algebra $\mathscr{A}$ has no nonzero commutative ideal, then any Finsler $\mathscr{A}$-module must be a Hilbert $C^{*}$-module. If $\mathscr{A}$ is the commutative $C^{*}$-algebra $C_{0}(X)$ of all continuous complexvalued functions vanishing at infinity on a locally compact Hausdorff space $X$, then any Finsler $\mathscr{A}$-module is isomorphic to the module of continuous sections of a bundle of Banach spaces over $X$. The concept of a $\varphi$-morphism between Finsler modules was introduced in [1].

The Gelfand-Naimark-Segal (GNS) representation theorem is one of the most useful theorems, which is applied in operator algebras and mathematical physics. That provides a procedure to construct representations of $C^{*}$-algebras. A generalization of GNS construction to a topological $*$-algebra established by Borchers, Uhlmann and Powers leading to unbounded $*$-representations of $*$-algebras; see [12]. Another is a generalization of a positive linear functional to a completely positive map studied by Stinespring [14], see also [6].

2010 Mathematics Subject Classification. Primary 46L08 Secondary 46L05.

Key words and phrases. Finsler module, quasi-representation, $\varphi$-morphism, nondegenerate quasirepresentation, irreducible quasi-representation.

* Corresponding author. 
Let $\mathscr{A}$ be a $C^{*}$-algebra and let $\mathscr{A}^{+}$denote the positive cone of all positive elements of $\mathscr{A}$. We define a Finsler $\mathscr{A}$-module to be a right $\mathscr{A}$-module $\mathscr{E}$ equipped with a map $\rho: \mathscr{E} \rightarrow \mathscr{A}^{+}$ (denoted by $\rho_{\mathscr{A}}$ if there is an ambiguity) satisfying the following conditions:

(i) The map $\|\cdot\|_{\mathscr{E}}: x \longmapsto\|\rho(x)\|$ makes $\mathscr{E}$ into a Banach space.

(ii) $\rho(x a)^{2}=a^{*} \rho(x)^{2} a$, for all $a \in \mathscr{A}$ and $x \in \mathscr{E}$.

A Finsler module $\mathscr{E}$ over a $C^{*}$-algebra $\mathscr{A}$ is said to be full if the linear span of $\left\{\rho(x)^{2}\right.$ : $x \in \mathscr{E}\}$ is dense in $\mathscr{A}$. For example, if $\mathscr{E}$ is a (full) Hilbert $C^{*}$-module over $\mathscr{A}$ (see [7]), then $\mathscr{E}$ together with $\rho(x)=\langle x, x\rangle^{\frac{1}{2}}$ is a (full) Finsler module over $\mathscr{A}$, since

$$
\rho(x a)^{2}=<x a, x a>=a^{*}<x, x>a=a^{*} \rho(x)^{2} a .
$$

In particular, every $C^{*}$-algebra $\mathscr{A}$ is a full Finsler module over $\mathscr{A}$ under the mapping $\rho(x)=$ $\left(x^{*} x\right)^{\frac{1}{2}}$.

Our goal is to extend the notion of a representation of a Hilbert $C^{*}$-module to the framework of Finsler $\mathscr{A}$-modules. We show that every Finsler $\mathscr{A}$-module has a quasirepresentation into the Banach space $\mathbb{B}(\mathscr{H}, \mathscr{K})$ of all bounded linear operators between some Hilbert spaces $\mathscr{H}$ and $\mathscr{K}$. We define the notion of completely positive $\varphi$-morphism and establish a Stinespring type theorem in the framework of Finsler modules over $C^{*}$ algebras. We also introduce the notions of the nondegeneracy and the irreducibility of quasi-representations and study some interrelations between them.

\section{QUASI-REPRESENTATIONS OF FINSLER MODULES}

We start our work by giving the definition of a $\varphi$-morphism of a Finsler module.

Definition 2.1. Suppose that $\left(\mathscr{E}, \rho_{\mathscr{A}}\right)$ and $\left(\mathscr{F}, \rho_{\mathscr{B}}\right)$ are Finsler modules over $C^{*}$-algebras $\mathscr{A}$ and $\mathscr{B}$, respectively and $\varphi: \mathscr{A} \rightarrow \mathscr{B}$ is a $*$-homomorphism of $C^{*}$-algebras. A (not necessarily linear) map $\Phi: \mathscr{E} \rightarrow \mathscr{F}$ is said to be a $\varphi$-morphism of Finsler modules if the following conditions are satisfied:

(i) $\rho_{\mathscr{B}}(\Phi(x))=\varphi\left(\rho_{\mathscr{A}}(x)\right)$;

(ii) $\Phi(x a)=\Phi(x) \varphi(a)$.

for all $x \in \mathscr{E}$ and $a \in \mathscr{A}$. In the case of Hilbert $C^{*}$-modules, $\Phi$ is assumed to be linear and then condition (ii) is deduced from (i).

Now we introduce the notion of a quasi-representation of a Finsler module. Due to $\mathbb{B}(\mathscr{H}, \mathscr{K})$ is a Hilbert $C^{*}$-module over $\mathbb{B}(\mathscr{H})$ via $\langle T, S\rangle=T^{*} S$, we can endow $\mathbb{B}(\mathscr{H}, \mathscr{K})$ a Finsler 
structure by

$$
\rho_{0}(T)=\left(T^{*} T\right)^{\frac{1}{2}}
$$

Definition 2.2. Let $(\mathscr{E}, \rho)$ be a Finsler module over a $C^{*}$-algebra $\mathscr{A}$. A map $\Phi: \mathscr{E} \rightarrow$ $\mathbb{B}(\mathscr{H}, \mathscr{K})$, where $\varphi: \mathscr{A} \rightarrow \mathbb{B}(\mathscr{H})$ is a representation of $\mathscr{A}$ is called a quasi-representation of $\mathscr{E}$ if $\rho_{0}(\Phi(x))=\varphi(\rho(x))$ for all $x \in \mathscr{E}$.

We are going to show that for every Finsler $\mathscr{A}$-module there is a quasi-representation to $\mathbb{B}(\mathscr{H}, \mathscr{K})$ for some Hilbert spaces $\mathscr{H}$ and $\mathscr{K}$, see also [9].

Theorem 2.3. Suppose $\mathscr{E}$ is a Finsler $\mathscr{A}$-module with the associated map $\rho: \mathscr{E} \rightarrow \mathscr{A}^{+}$. Then there is a quasi-representation $\Phi: \mathscr{E} \rightarrow \mathbb{B}(\mathscr{H}, \mathscr{K})$ for some Hilbert spaces $\mathscr{H}$ and $\mathscr{K}$.

Proof. By the Gelfand-Naimark theorem for $C^{*}$-algebras, there is a representation $\varphi: \mathscr{A} \rightarrow$ $\mathbb{B}(\mathscr{H})$ for some Hilbert space $\mathscr{H}$. We want to construct a Hilbert space $\mathscr{K}$. Put

$$
\mathscr{K}_{0}:=\operatorname{span}\{\varphi(a) f: a \in \mathscr{A}, f: \mathscr{E} \rightarrow \mathscr{H} \text { is a map with a finite support }\}
$$

and define on $\mathscr{K}_{0}$ an inner product by

$$
\langle\varphi(a) f, \varphi(b) g\rangle=\sum_{x \in \mathscr{E}}\langle\varphi(a) f(x), \varphi(b) g(x)\rangle .
$$

Note that if $\left\langle\sum_{i=1}^{n} \varphi\left(a_{i}\right) f_{i}, \sum_{i=1}^{n} \varphi\left(a_{i}\right) f_{i}\right\rangle=0$, then

$$
\sum_{x \in \mathscr{E}}\left\langle\sum_{i=1}^{n} \varphi\left(a_{i}\right) f_{i}(x), \sum_{i=1}^{n} \varphi\left(a_{i}\right) f_{i}(x)\right\rangle=0 .
$$

Thus $\sum_{i=1}^{n} \varphi\left(a_{i}\right) f_{i}(x)=0$ for each $x \in \mathscr{E}$, whence $\sum_{i=1}^{n} \varphi\left(a_{i}\right) f_{i}=0$.

Let us consider the closure $\overline{\mathscr{K}_{0}}$ of $\mathscr{K}_{0}$ to get a Hilbert space, which is denoted by $\mathscr{K}$. For any $y \in \mathscr{E}$ and $h \in \mathscr{H}$, the map $h_{y}: \mathscr{E} \rightarrow \mathscr{H}$ defined by

$$
h_{y}(x)= \begin{cases}h & x=y \\ 0 & x \neq y\end{cases}
$$

has a finite support. For $x \in \mathscr{E}$, define $\Phi(x): \mathscr{H} \rightarrow \mathscr{K}$ by $\Phi(x) h=\varphi(\rho(x)) h_{x}$. We show that $\Phi(x) \in \mathbb{B}(\mathscr{H}, \mathscr{K})$. Clearly $\Phi(x)$ is linear. Also $\Phi(x)$ is bounded, since

$$
\begin{aligned}
\|\Phi(x) h\|^{2} & =\langle\Phi(x) h, \Phi(x) h\rangle=\left\langle\varphi(\rho(x)) h_{x}, \varphi(\rho(x)) h_{x}\right\rangle \\
& =\sum_{y \in \mathscr{E}}\left\langle\varphi(\rho(x)) h_{x}(y), \varphi(\rho(x)) h_{x}(y)\right\rangle=\langle\varphi(\rho(x)) h, \varphi(\rho(x)) h\rangle \\
& \leq\|\varphi(\rho(x))\|^{2}\|h\|^{2},
\end{aligned}
$$


whence $\|\Phi(x)\| \leq\|\varphi(\rho(x))\|$.

Further,

$$
\begin{aligned}
\left\langle\Phi(x)^{*} \Phi(x) h, h^{\prime}\right\rangle & =\left\langle\Phi(x) h, \Phi(x) h^{\prime}\right\rangle=\left\langle\varphi(\rho(x)) h_{x}, \varphi(\rho(x)) h_{x}^{\prime}\right\rangle \\
& =\sum_{y \in \mathscr{E}}\left\langle\varphi(\rho(x)) h_{x}(y), \varphi(\rho(x)) h_{x}^{\prime}(y)\right\rangle \\
& =\left\langle\varphi(\rho(x)) h, \varphi(\rho(x)) h^{\prime}\right\rangle=\left\langle\varphi\left(\rho(x)^{2}\right) h, h^{\prime}\right\rangle,
\end{aligned}
$$

for all $h, h^{\prime} \in \mathscr{H}$ and $x \in \mathscr{E}$. Hence $\Phi(x)^{*} \Phi(x)=\varphi\left(\rho(x)^{2}\right)$. Hence

$$
\left(\Phi(x)^{*} \Phi(x)\right)^{\frac{1}{2}}=\varphi(\rho(x)) .
$$

It follows from (2.1) and equality (2.2) that $\rho_{0}(\Phi(x))=\varphi(\rho(x))$.

Remark 2.4. If $\Phi$ is surjective and $\mathbb{B}(\mathscr{H}, \mathscr{K})$ is a full Finsler $\mathbb{B}(\mathscr{H})$-module, then by $[1$, Theorem 3.4(iv)], $\varphi$ is surjective.

In the next section the notion of completely positive $\varphi$-morphism is introduced and a construction of Stinespring's theorem for Finsler modules is given.

\section{A STINESPRING TYPE THEOREM FOR FINSLER MODULES}

The Stinespring theorem was first introduced in the work of Stinespring in 1995 that described the structure of completely positive maps of a $C^{*}$-algebra into the $C^{*}$-algebra of all bounded linear operators on a Hilbert space; see [14]. Recently Asadi [3] proved this theorem for Hilbert $C^{*}$-modules. Further, Bhat et al. [4] improve the result of [3] with omitting a technical condition. In this section we intend to establish a Stinespring type theorem in the framework of Finsler modules over $C^{*}$-algebras.

A $\varphi$-morphism $\Phi: \mathscr{E} \rightarrow \mathbb{B}(\mathscr{H}, \mathscr{K})$ is called completely positive if the map $\varphi: \mathscr{A} \rightarrow \mathbb{B}(\mathscr{H})$ is completely positive.

Theorem 3.1. Let $(\mathscr{E}, \rho)$ be a Finsler module over a unital $C^{*}$-algebra $\mathscr{A}$, let $\mathscr{H}, \mathscr{K}$ be Hilbert spaces and let $\Phi: \mathscr{E} \rightarrow \mathbb{B}(\mathscr{H}, \mathscr{K})$ be a completely positive map associated to a completely positive map $\varphi: \mathscr{A} \rightarrow \mathbb{B}(\mathscr{H})$. Then there exist Hilbert spaces $\mathscr{H}^{\prime}, \mathscr{K}^{\prime}$ and isometries $V: \mathscr{H} \rightarrow \mathscr{H}^{\prime}, W: \mathscr{K} \rightarrow \mathscr{K}^{\prime}$, a $*$-homomorphism $\theta: \mathscr{A} \rightarrow \mathbb{B}\left(\mathscr{H}^{\prime}\right)$ and a $\theta$-morphism $\Psi: \mathscr{E} \rightarrow \mathbb{B}\left(\mathscr{H}^{\prime}, \mathscr{K}^{\prime}\right)$ such that $\varphi(a)=V^{*} \theta(a) V, \Phi(x)=W^{*} \Psi(x) V$ for all $x \in \mathscr{E}$ and $a \in \mathscr{A}$.

Proof. By [11, Theorem 4.1] there exist a Hilbert space $\mathscr{H}^{\prime}=\mathscr{A} \otimes \mathscr{H}$, a representation $\theta: \mathscr{A} \rightarrow \mathbb{B}\left(\mathscr{H}^{\prime}\right)$ and an isometry $V: \mathscr{H} \rightarrow \mathscr{H}^{\prime}$ defined by $V(h)=1 \otimes h$ such that 
$\varphi(a)=V^{*} \theta(a) V$. We may consider a minimal Stinespring representation for $\theta$, where $\mathscr{H}^{\prime}$ is the closed linear span of $\{\theta(a) V h: a \in \mathscr{A}, h \in \mathscr{H}\}$.

Now, we put $\mathscr{K}^{\prime}$ to be the closed linear span of $\{\Phi(x) h: x \in \mathscr{E}, h \in \mathscr{H}\}$ and define the mapping $\Psi: \mathscr{E} \rightarrow \mathbb{B}\left(\mathscr{H}^{\prime}, \mathscr{K}^{\prime}\right), x \mapsto \Psi(x)$, where $\Psi(x): \operatorname{span}\{\theta(a) V h, a \in \mathscr{A}, h \in \mathscr{H}\} \rightarrow$ $\mathscr{K}^{\prime}$ is defined by $\Psi(x)\left(\sum_{i=1}^{n} \theta\left(a_{i}\right) V h_{i}\right)=\sum_{i=1}^{n} \Phi\left(x a_{i}\right) h_{i}$ for $x \in \mathscr{E}, a_{i} \in \mathscr{A}, h_{i} \in \mathscr{H}$.

The map $\Psi(x)$ is well-defined and bounded, since

$$
\begin{aligned}
\left\|\Psi(x)\left(\sum_{i=1}^{n} \theta\left(a_{i}\right) V h_{i}\right)\right\|^{2} & =\left\|\sum_{i=1}^{n} \Phi\left(x a_{i}\right) h_{i}\right\|^{2} \\
& =\sum_{i, j=1}^{n}\left\langle\Phi\left(x a_{j}\right)^{*} \Phi\left(x a_{i}\right) h_{i}, h_{j}\right\rangle \\
& =\sum_{i, j=1}^{n}\left\langle\varphi\left(a_{j}^{*}\right) \Phi(x)^{*} \Phi(x) \varphi\left(a_{i}\right) h_{i}, h_{j}\right\rangle \\
& =\sum_{i, j=1}^{n}\left\langle\varphi\left(a_{j}^{*}\right) \varphi\left(\rho(x)^{2}\right) \varphi\left(a_{i}\right) h_{i}, h_{j}\right\rangle \\
& =\sum_{i, j=1}^{n}\left\langle\varphi\left(a_{j}^{*} \rho(x)^{2} a_{i}\right) h_{i}, h_{j}\right\rangle \\
& =\sum_{i, j=1}^{n}\left\langle V^{*} \theta\left(a_{j}^{*} \rho(x)^{2} a_{i}\right) V h_{i}, h_{j}\right\rangle \\
& =\sum_{i, j=1}^{n}\left\langle\theta\left(\rho(x)^{2}\right) \theta\left(a_{i}\right) V h_{i}, \theta\left(a_{j}\right) V h_{j}\right\rangle \\
& \leq\left\|\theta\left(\rho(x)^{2}\right)\right\|\left\|\sum_{i=1}^{n} \theta\left(a_{i}\right) V h_{i}\right\|^{2} \\
& \leq\|\rho(x)\|^{2}\left\|\sum_{i=1}^{n} \theta\left(a_{i}\right) V h_{i}\right\|^{2} \\
& =\|x\|^{2}\left\|\sum_{i=1}^{n} \theta\left(a_{i}\right) V h_{i}\right\|^{2} .
\end{aligned}
$$


The mapping $\Psi$ is a $\theta$-morphism, since for all $a, b \in \mathscr{A}$ and $h, g \in \mathscr{H}$

$$
\begin{aligned}
\left\langle\Psi(x)^{*} \Psi(x)(\theta(a) V h), \theta(b) V g\right\rangle & =\langle\Psi(x)(\theta(a) V h), \Psi(x)(\theta(b) V g)\rangle \\
& =\langle\Phi(x a) h, \Phi(x b) g\rangle \\
& =\langle\Phi(x) \varphi(a) h, \Phi(x) \varphi(b) g\rangle \\
& =\left\langle\Phi(x)^{*} \Phi(x) \varphi(a) h, \varphi(b) g\right\rangle \\
& =\left\langle\varphi\left(\rho(x)^{2}\right) \varphi(a) h, \varphi(b) g\right\rangle \\
& =\left\langle\varphi\left(b^{*} \rho(x)^{2} a\right) h, g\right\rangle \\
& =\left\langle V^{*} \theta\left(b^{*} \rho(x)^{2} a\right) V h, g\right\rangle \\
& =\left\langle\theta\left(\rho(x)^{2}\right) \theta(a) V h, \theta(b) V g\right\rangle
\end{aligned}
$$

whence $\Psi(x)^{*} \Psi(x)=\theta\left(\rho(x)^{2}\right)$. Moreover

$$
\begin{aligned}
\Psi(x) \theta(a)(\theta(b) V h) & =\Psi(x)(\theta(a b) V h) \\
& =\Phi(x(a b)) h \\
& =\Phi((x a) b) h \\
& =\Psi(x a)(\theta(b) V h),
\end{aligned}
$$

so that $\Psi(x) \theta(a)=\Psi(x a)$.

Since $\mathscr{K}^{\prime} \subseteq \mathscr{K}$ we can consider a map $W$ as the orthogonal projection of $\mathscr{K}$ onto $\mathscr{K}^{\prime}$. Hence $W^{*}: \mathscr{K}^{\prime} \rightarrow \mathscr{K}$ is the inclusion map, whence for any $k^{\prime} \in \mathscr{K}^{\prime}$ we have $W W^{*}\left(k^{\prime}\right)=$ $W\left(k^{\prime}\right)=k^{\prime}$, that is $W W^{*}=I_{\mathscr{K}^{\prime}}$.

Finally we observe that $W^{*} \Psi(x) V h=\Psi(x) V h=\Psi(x)(\theta(1) V h)=\Phi(x) h$, that is $W^{*} \Psi(x) V=$ $\Phi(x)$.

\section{NONDEGENERATE AND IRREDUCIBLE QUASI-REPRESENTATIONS}

In this section we define the notions of nondegenerate and irreducible quasi-representations of Finsler modules and describe relations between the nondegeneracy and the irreduciblity, see [2]. Throughout this section we assume that the quasi-representations satisfy condition (ii) of Definition 2.1.

Definition 4.1. Let $\Phi: \mathscr{E} \rightarrow \mathbb{B}(\mathscr{H}, \mathscr{K})$ be a quasi-representation of a Finsler module $\mathscr{E}$ over a $C^{*}$-algebra $\mathscr{A}$. The map $\Phi$ is said to be nondegenerate if $\overline{\Phi(\mathscr{E}) \mathscr{H}}=\mathscr{K}$ and $\overline{\Phi(\mathscr{E})^{*} \mathscr{K}}=\mathscr{H}$ (or equivalently, if there exist $\xi \in \mathscr{H}, \eta \in \mathscr{K}$ such that $\Phi(\mathscr{E}) \xi=0$ and 
$\Phi(\mathscr{E})^{*} \eta=0$, then $\left.\xi=\eta=0\right)$. Recall that a representation $\varphi: \mathscr{A} \rightarrow \mathbb{B}(\mathscr{H})$ of a $C^{*}$-algebra $\mathscr{A}$ is nondegenerate if $\overline{\varphi(\mathscr{A}) \mathscr{H}}=\mathscr{H}$ (or equivalently, if there exists $\xi \in \mathscr{H}$ such that $\varphi(\mathscr{A}) \xi=0$, then $\xi=0$ ), see [13, definition A.1.].

Theorem 4.2. If $\Phi: \mathscr{E} \rightarrow \mathbb{B}(\mathscr{H}, \mathscr{K})$ is a nondegenerate quasi-representation, then $\varphi:$ $\mathscr{A} \rightarrow \mathbb{B}(\mathscr{H})$ is a nondegenerate representation. If $\mathscr{E}$ is full and $\varphi$ is nondegenerate, then $\Phi$ is also nondegenerate.

Proof. Suppose that $\Phi$ is nondegenerate and $\varphi(\mathscr{A}) \xi=0$. It follows from the Hewitt-Cohen factorization theorem that $\Phi(\mathscr{E}) \xi=\Phi(\mathscr{E} \mathscr{A}) \xi=\Phi(\mathscr{E}) \varphi(\mathscr{A}) \xi=0$. We conclude that $\xi=0$. Thus $\varphi$ is nondegenerate.

Suppose that $\Phi(\mathscr{E}) \xi=0$ for some $\xi \in \mathscr{H}$. Then for any $x \in \mathscr{E}$ we have $\|\Phi(x) \xi\|^{2}=$ $\left\langle\Phi(x)^{*} \Phi(x) \xi, \xi\right\rangle=\left\langle\varphi\left(\rho(x)^{2}\right) \xi, \xi\right\rangle=\|\varphi(\rho(x))\|^{2}=0$. Since $\mathscr{E}$ is a full Finsler $\mathscr{A}$-module, $a=\lim _{n \rightarrow \infty} \sum_{i=1}^{k_{n}} \lambda_{i, n} \rho\left(x_{i, n}\right)^{2}$ for some $k_{n} \in \mathbb{N}, x_{i, n} \in \mathscr{E}$ and $\lambda_{i, n} \in \mathbb{C}$. Hence

$$
\varphi(a) \xi=\lim _{n \rightarrow \infty} \sum_{i=1}^{k_{n}} \lambda_{i, n} \varphi\left(\rho\left(x_{i, n}\right)\right)^{2} \xi=\lim _{n \rightarrow \infty} \sum_{i=1}^{k_{n}} \lambda_{i, n} \varphi\left(\rho\left(x_{i, n}\right)\right) \varphi\left(\rho\left(x_{i, n}\right)\right) \xi=0,
$$

whence $\xi=0$.

Remark 4.3. The second result of Theorem 4.2 may fail, if the condition of being full is dropped. To see this take $\mathscr{A}$ to be a nondegenerate von Neumann algebra acting on a Hilbert space, which has a nontrivial central projection $P$. Hence the identity map $\varphi: \mathscr{A} \rightarrow \mathbb{B}(\mathscr{H})$ is assumed to be nondegenerate.

Put $\mathscr{E}=\mathscr{A} P=\{a P: a \in \mathscr{A}\}$ as a Finsler $\mathscr{A}$-module equipped with $\rho(a P)=|a P|$. Clearly $\mathscr{A} P$ is not full. The identity map $\Phi: \mathscr{A} P \rightarrow \mathbb{B}(\mathscr{H})$ satisfies the following:

(i) $\rho_{0} \Phi(a P)=\rho_{0}(a P)=|a P|=\varphi(|a P|)=\varphi \rho(a P)$, where $\rho_{0}$ is defined as in (2.1).

(ii) $\Phi(a P b)=\Phi(a P) \varphi(b)$ for all $b \in \mathscr{A}$.

Hence $\Phi$ is a quasi-representation of $\mathscr{E}$, which is not clearly nondegenerate, since

$$
\overline{\Phi(\mathscr{E}) \mathscr{H}}=\overline{\mathscr{A} P(\mathscr{H})}=\overline{P(\mathscr{A} \mathscr{H})} \subseteq \overline{P(\mathscr{H})}=P(\mathscr{H}) \neq \mathscr{H}
$$

In the following corollary we investigate a condition under which the representation $\varphi$ and the quasi-representation $\Phi$ are nondegenerate.

Corollary 4.4. If $\varphi(\rho(x))=I_{\mathscr{H}}$, then both $\Phi$ and $\varphi$ are nondegenerate. 
Proof. Suppose $\Phi(\mathscr{E}) \xi=0$ for some $\xi \in \mathscr{H}$. Then for all $x \in \mathscr{E}$ we have $\|\Phi(x) \xi\|^{2}=$ $\left\langle\Phi(x)^{*} \Phi(x) \xi, \xi\right\rangle=\left\langle\varphi\left(\rho(x)^{2}\right) \xi, \xi\right\rangle=\|\xi\|^{2}=0$, so that $\xi=0$. The nondegeneracy of $\varphi$ follows from Theorem 4.2.

Definition 4.5. Let $\Phi: \mathscr{E} \rightarrow B\left(\mathscr{H}, \mathscr{H}^{\prime}\right)$ be a quasi-representation of a Finsler module $\mathscr{E}$ over a $C^{*}$-algebra $\mathscr{A}$ and let $\mathscr{K}, \mathscr{K}^{\prime}$ be closed subspaces of $\mathscr{H}$ and $\mathscr{H}^{\prime}$, respectively. A pair of subspaces $\left(\mathscr{K}, \mathscr{K}^{\prime}\right)$ is said to be $\Phi$-invariant if $\Phi(\mathscr{E}) \mathscr{K} \subseteq \mathscr{K}^{\prime}$ and $\Phi(\mathscr{E})^{*} \mathscr{K}^{\prime} \subseteq \mathscr{K}$. The quasi-representation $\Phi$ is said to be irreducible if $(0,0)$ and $\left(\mathscr{H}, \mathscr{H}^{\prime}\right)$ are the only $\Phi$-invariant pairs. Recall that a representation $\varphi: \mathscr{A} \rightarrow \mathbb{B}(\mathscr{H})$ of a $C^{*}$-algebra $\mathscr{A}$ is irreducible if 0 and $\mathscr{H}$ are only closed subspaces of $\mathscr{H}$ being $\varphi$-invariant, i.e. are invariant for $\varphi(\mathscr{A})$.

Theorem 4.6. Suppose that the quasi-representation $\Phi: \mathscr{E} \rightarrow \mathbb{B}(\mathscr{H}, \mathscr{K})$ constructed in Theorem 2.3 is irreducible. Then so is $\varphi: \mathscr{A} \rightarrow \mathbb{B}(\mathscr{H})$. If $\mathscr{E}$ is full and $\varphi$ is irreducible, then $\Phi$ is irreducible.

Proof. Suppose that $\Phi$ is irreducible and a closed subspace $\mathscr{K}$ of $\mathscr{H}$ is $\varphi$-invariant. Consider $\mathscr{K}^{\prime}=\overline{\Phi(\mathscr{E}) \mathscr{K}}$. Clearly $\Phi(\mathscr{E}) \mathscr{K} \subseteq \mathscr{K}^{\prime}$. Due to $\overline{\varphi(\mathscr{A}) \mathscr{K}} \subseteq \mathscr{K}$ we observe that $\overline{\varphi\left(\rho(x)^{2}\right) \mathscr{K}} \subseteq \mathscr{K}$, whence $\overline{\Phi(x)^{*} \Phi(x) \mathscr{K}} \subseteq \mathscr{K}$ for all $x \in \mathscr{E}$. Now let $x \neq y$. In the notation of Theorem 2.3 we have

$$
\begin{aligned}
\left\langle\Phi(x)^{*} \Phi(y) h, h^{\prime}\right\rangle & =\left\langle\Phi(y) h, \Phi(x) h^{\prime}\right\rangle=\left\langle\varphi(\rho(y)) h_{y}, \varphi(\rho(x)) h_{x}^{\prime}\right\rangle \\
& =\sum_{z \in \mathscr{E}}\left\langle\varphi(\rho(y)) h_{y}(z), \varphi(\rho(x)) h_{x}^{\prime}(z)\right\rangle=0,
\end{aligned}
$$

for all $h, h^{\prime} \in \mathscr{H}$. Put $h^{\prime}=\Phi(x)^{*} \Phi(y) h$ to get $\left\langle\Phi(x)^{*} \Phi(y) h, \Phi(x)^{*} \Phi(y) h\right\rangle=0$. So that $\Phi(x)^{*} \Phi(y) h=0$. Therefore $\Phi(x)^{*} \Phi(y) \mathscr{K}=0 \mathscr{K} \subseteq \mathscr{K}$. It follows that $\Phi(E)^{*} \overline{\Phi(E) \mathscr{K} \subseteq}$ $\overline{\Phi(E)^{*} \Phi(E) \mathscr{K}} \subseteq \mathscr{K}$. Since $\Phi$ is irreducible, we conclude that $\left(\mathscr{K}, \mathscr{K}^{\prime}\right)=(0,0)$ or $\left(\mathscr{K}, \mathscr{K}^{\prime}\right)=$ $\left(\mathscr{H}, \mathscr{H}^{\prime}\right)$, hence $\mathscr{K}=0$ or $\mathscr{K}=\mathscr{H}$. This implies that $\varphi$ is irreducible.

Now assume that $\varphi$ is irreducible. It follows from [8, Remark 4.1.4] that $\varphi$ is nondegenerate. By Theorem 4.2, $\Phi$ is nondegenerate.

Consider $\left(\mathscr{K}, \mathscr{K}^{\prime}\right)$ as a $\Phi$-invariant pair of subspaces. Any $a \in \mathscr{A}$ can be represented as $a=\lim _{n \rightarrow \infty} \sum_{i=1}^{k_{n}} \lambda_{i, n} \rho\left(x_{i, n}\right)^{2}$ for some $k_{n} \in \mathbb{N}, x_{i, n} \in \mathscr{E}$ and $\lambda_{i, n} \in \mathbb{C}$. Hence

$$
\varphi(a) \mathscr{K}=\lim _{n \rightarrow \infty} \sum_{i=1}^{k_{n}} \lambda_{i, n} \varphi\left(\rho\left(x_{i, n}\right)\right)^{2} \mathscr{K}=\lim _{n \rightarrow \infty} \sum_{i=1}^{k_{n}} \lambda_{i, n} \Phi\left(x_{i, n}\right)^{*} \Phi\left(x_{i, n}\right) \mathscr{K} \subseteq \mathscr{K},
$$

Hence $\mathscr{K}=0$ or $\mathscr{K}=\mathscr{H}$.

If $\mathscr{K}=0$ then $\Phi(\mathscr{E})^{*} \mathscr{K}^{\prime} \subseteq \mathscr{K}=0$, and for every $\xi^{\prime} \in \mathscr{K}^{\prime}$ we have $0=\left\langle\Phi(x)^{*} \xi^{\prime}, \xi\right\rangle=$ 
$\left\langle\xi^{\prime}, \Phi(x) \xi\right\rangle$ for $x \in \mathscr{E}$ and $\xi \in \mathscr{H}$, so that $\mathscr{K}^{\prime} \perp \overline{\Phi(\mathscr{E}) \mathscr{H}}=\mathscr{H}^{\prime}$. Since $\mathscr{K}^{\prime} \subseteq \mathscr{H}^{\prime}$, we have $\mathscr{K}^{\prime}=0$.

If $\mathscr{K}=\mathscr{H}$, then $\mathscr{H}^{\prime}=\overline{\Phi(\mathscr{E}) \mathscr{H}}=\overline{\Phi(\mathscr{E}) \mathscr{K}} \subseteq \mathscr{K}^{\prime}$. Hence $\mathscr{K}^{\prime}=\mathscr{H}^{\prime}$. Therefore $\Phi$ is irreducible.

Remark 4.7. The result may fail, if the condition of being full is dropped. The closed subspace $P(\mathscr{H})$ in Remark 4.3 when $\varphi: \mathscr{A} \rightarrow \mathbb{B}(\mathscr{H})$ is irreducible provides a counterexample.

Next we present some conditions under which the quasi-representation $\Phi$ is nondegenerate and irreducible.

Corollary 4.8. Let $\mathscr{E}$ be a full Finsler $\mathscr{A}$-module and let $\varphi: \mathscr{A} \rightarrow \mathbb{B}(\mathscr{H})$ is irreducible. Then the quasi-representation $\Phi: \mathscr{E} \rightarrow \mathbb{B}(\mathscr{H}, \mathscr{K})$ is nondegenerate and irreducible.

Proof. Since $\varphi$ is irreducible, it is nondegenerate. Since $\mathscr{E}$ is full, by Theorem $4.2, \Phi$ is nondegenerate and by Theorem 4.6, $\Phi$ is irreducible.

Theorem 4.9. Let $\mathscr{E}$ be a full Finsler $\mathscr{A}$-module. Then $\Phi(\mathscr{E})$ is a subset of the space $\mathbb{K}\left(\mathscr{H}, \mathscr{H}^{\prime}\right)$ of all compact operators from $\mathscr{H}$ into $\mathscr{H}^{\prime}$ if and only if $\varphi(\mathscr{A}) \subseteq \mathbb{K}(\mathscr{H})$.

Proof. Suppose $\varphi(\mathscr{A}) \subseteq \mathbb{K}(\mathscr{H})$. Applying the Hewitt-Cohen factorization theorem we have $\Phi(\mathscr{E})=\Phi(\mathscr{E} \mathscr{A})=\Phi(\mathscr{E}) \varphi(\mathscr{A}) \subseteq \mathbb{K}\left(\mathscr{H}, \mathscr{H}^{\prime}\right)$.

Conversely, suppose that $\Phi(\mathscr{E}) \subseteq \mathbb{K}\left(\mathscr{H}, \mathscr{H}^{\prime}\right)$. Since $\mathscr{E}$ is full we have

$$
\varphi(a)=\lim _{n \rightarrow \infty} \sum_{i=1}^{k_{n}} \lambda_{i, n} \varphi\left(\rho\left(x_{i, n}\right)\right)^{2}=\lim _{n \rightarrow \infty} \sum_{i=1}^{k_{n}} \lambda_{i, n} \Phi\left(x_{i, n}\right)^{*} \Phi\left(x_{i, n}\right) \in \mathbb{K}(\mathscr{H}),
$$

where $a=\lim _{n \rightarrow \infty} \sum_{i=1}^{k_{n}} \lambda_{i, n} \rho\left(x_{i, n}\right)^{2}$ for some $k_{n} \in \mathbb{N}, x_{i, n} \in \mathscr{E}$ and $\lambda_{i, n} \in \mathbb{C}$.

Now in the next two examples we illustrate the considered situations in the notation of Theorem 2.3.

Example 4.10. By [5, Theorem 1.10.2] the identity map $\varphi: \mathbb{K}(\mathscr{H}) \rightarrow \mathbb{B}(\mathscr{H})$ is irreducible. It is known that the $C^{*}$-algebra $\mathbb{K}(\mathscr{H})$ is a full Finsler module over $\mathbb{K}(\mathscr{H})$ with $\rho(T)=|T|$. Hence the quasi-representation $\Phi: \mathbb{K}(\mathscr{H}) \rightarrow \mathbb{B}(\mathscr{H}, \mathscr{K})$ is nondegenerate and irreducible.

Example 4.11. Consider $\varphi=I: \mathbb{B}(\mathscr{H}) \rightarrow \mathbb{B}(\mathscr{H})$. Then $\varphi(\mathbb{B}(\mathscr{H}))^{c}=\{T \in \mathbb{B}(\mathscr{H}) ; \varphi(S) T=$ $T \varphi(S)$, for all $S \in \mathbb{B}(\mathscr{H})\}=\{T \in \mathbb{B}(\mathscr{H}) ; S T=T S$, for all $S \in \mathbb{B}(\mathscr{H})\}=\mathbb{C} I$. Hence $\varphi$ is irreducible. Also $\mathbb{B}(\mathscr{H})$ is a full Finsler $\mathbb{B}(\mathscr{H})$-module, so that the quasi-representation $\Phi: \mathbb{B}(\mathscr{H}) \rightarrow \mathbb{B}(\mathscr{H}, \mathscr{K})$ is nondegenerate and irreducible. 
Acknowledgement. The authors would like to sincerely thank Professor M. Joiţa for some useful comments improving the paper.

\section{REFERENCES}

[1] M. Amyari, A. Niknam, On homomorphisms of Finsler modules, Inter. Math. J. 3 (2003), 277-281.

[2] LJ. Arambašić, Irreducible representations of Hilbert $C^{*}$-modules, Math. Proc. R. Ir. Acad. 105A (2005), no. 2, 11-24.

[3] M.B. Asadi, Stinespring's theorem for Hilbert $C^{*}$-modules, J. Operator Theory 62 (2008), no. 2, $235-238$.

[4] B.V.R. Bhat, G. Ramesh, K. Sumsh, Stinespring's theorem for maps on Hilbert $C^{*}$-modules, $J$. Operator Theory, to appear.

[5] K.R. Davidson, $C^{*}$-algebra by Example, Fields Institute Monographs, 6, Amer. Math. Soc., Providence. RI, 1996.

[6] M. JorŢA, Strict completely positive maps between locally $C^{*}$-algebras and representations on Hilbert modules, J. London Math. Soc. (2) 66 (2002), no. 2, 421-432.

[7] V.M. Manuilov, E.V. Troitsky, Hilbert $C^{*}$-modules. Translated from the 2001 Russian original by the authors. Translations of Mathematical Monographs, 226. American Mathematical Society, Providence, RI, 2005.

[8] G.J. Murphy, $C^{*}$-Algebras and Operator Theory, Academic press, New York, 1990.

[9] G.J. Murphy, Positive Definite Kernels and Hilbert $C^{*}$-Modules, Proc. Edinburgh Math. Soc. 40(2)(1997), 367-374.

[10] N.C. Phillips, N. Weaver, Modules with norms which take values in a $C^{*}$-algebra, Pacific J. Math. 185 (1998), no. 1, 163-181.

[11] V. PAulsen, Completely bounded maps and operator algebras, Cambridge studies in Advanced Mathematics, vol. 78, Cambridge University Press, Cambridge, 2002.

[12] R.T. Powers, Self-adjoint algebras of unbounded operators, Commun. Math. Phys. 21 (1971), 85-124.

[13] M. Skeide, Generalised matrix $C^{*}$-algebras and representation of Hilbert modules, Math. Proc. R. Ir. Acad. 100 A (2000), no. 1, 11-38.

[14] W.F. Stinespring, Positive functions on $C^{*}$-algebras, Proc. Amer. Math. Sci. 6 (1955), 211-216.

1 Department of Mathematics, Faculty of science, Islamic Azad University-Mashhad Branch, Mashhad 91735, Iran.

E-mail address: amyari@mshdiau.ac.ir and Maryam_Amyari@yahoo.com

E-mail address: m-chakoshi@mshdiau.ac.ir

2 Department of Pure Mathematics, Center of Excellence in Analysis on Algebraic Structures (CEAAS), Ferdowsi University of Mashhad, P.O. Box 1159, Mashhad 91775, Iran.

E-mail address: moslehian@ferdowsi.um.ac.ir and moslehian@member.ams.org 